\title{
Bacterial Quorum Sensing Signals Promote Large-Scale Remodeling of Lipid Membranes
}

Curran G. Gahan, ${ }^{1}$ Samarthaben J. Patel, ${ }^{1}$ Lawrence M. Chen, ${ }^{1}$ Daniel E. Manson, ${ }^{2}$ Zachary J. Ehmer, ${ }^{1}$ Helen E. Blackwell, ${ }^{2,}$ Reid C. Van Lehn, ${ }^{1, *}$ and David M. Lynn ${ }^{1,2, *}$

${ }^{1}$ Dept. of Chemical and Biological Engineering, Univ. of Wisconsin-Madison, 1415 Engineering Dr., Madison, WI 53706; ${ }^{2}$ Dept. of Chemistry, Univ. of Wisconsin-Madison, 1101 University Ave., Madison, WI 53706, USA; Email: (H.E.B.) blackwell@chem.wisc.edu; (R.C.V.) vanlehn@wisc.edu; (D.M.L.)dlynn@engr.wisc.edu

\section{Supporting Information}

\section{Supplementary Videos}

Video S1: A representative time-lapse fluorescence microscopy video of the formation of a SLB by vesicle fusion (VF). In this video, a suspension of large unilamellar vesicles at $0.1 \mathrm{mg} / \mathrm{mL}$ is introduced to a glass substrate in a flow cell and allowed to adsorb to the surface over time, eventually fusing to form a continuous, fluid SLB. The scale bar represents $30 \mu \mathrm{m}$.

Video S2: A representative time-lapse fluorescence microscopy video of a qualitative FRAP experiment on the SLB formed in Video S1. In this video, a spot on the bilayer formed in Video S1 was photobleached using the light source described in the Methods section, and the fluorescence intensity of that area was monitored over time to ensure that a fluid SLB was formed. The scale bar represents $30 \mu \mathrm{m}$.

Video S3: A time-lapse fluorescence microscopy experiment characterizing the influence of GML on an SLB formed by VF. In this video, a $250 \mu \mathrm{M}$ GML solution was added to a fluorescently labeled DOPC SLB, leading to the formation of hemispherical caps on the surface of the bilayer. The scale bar represents $30 \mu \mathrm{m}$.

Video S4: A representative time-lapse fluorescence microscopy video of the formation of a SLB by bicelle fusion. In this video, a suspension of bicelles at $0.062 \mathrm{mM}$ as introduced to a glass substrate in a flow cell and allowed to adsorb to the surface over time, eventually fusing to form a continuous, fluid SLB. The scale bar represents $30 \mu \mathrm{m}$.

Video S5: A representative time-lapse fluorescence microscopy video of a qualitative FRAP experiment on the SLB formed in Video S4. In this video, a spot in the bilayer formed in Video S4 was photobleached using the light source described in the Methods section, and the fluorescence intensity of that area was monitored to ensure that a fluid SLB was formed. The scale bar represents $30 \mu \mathrm{m}$.

Video S6: A representative time-lapse fluorescence microscopy experiment characterizing the influence of $200 \mathrm{uM} 3$-oxo-C12-HS on an SLB formed with VF. In this video, a $200 \mu \mathrm{M} 3$-oxoC12-HS solution was added to a fluorescently labeled DOPC SLB, leading to the formation of extended tubules on the surface of the bilayer. The scale bar represents $30 \mu \mathrm{m}$. 
Video S7: A representative time-lapse fluorescence microscopy experiment characterizing the influence of $200 \mathrm{uM} 3-0 x 0-\mathrm{C} 12-\mathrm{AHL}$ on an SLB formed with VF. In this video, a $200 \mu \mathrm{M} 3-$ oxo-C12-AHL solution was added to a fluorescently labeled DOPC SLB formed by vesicle fusion, leading to the formation of extended tubule structures, some of which collapsed into hemispherical caps on the surface of the bilayer. The scale bar represents $30 \mu \mathrm{m}$.

Video S8: A representative time-lapse fluorescence microscopy experiment characterizing the influence of $200 \mathrm{uM} 3$-oxo-C12-AHL on an SLB formed by bicelle formation. In this video, a $200 \mu \mathrm{M}$ 3-oxo-C12-AHL solution was added to a fluorescently labeled DOPC SLB formed by bicelle formation, leading to the formation of extended tubule structures, some of which collapsed into hemispherical caps on the surface of the bilayer; this result is consistent with that observed in Video S7, and suggests no significant difference in the behaviors of SLBs formed using vesicle fusion or bicelle formation upon the introduction of the amphiphiles studied here. The scale bar represents $30 \mu \mathrm{m}$.

Note: Each individual frame of the above videos is taken five seconds apart and six images are shown per second. Therefore, all the videos above are sped up 30x from "real time". 

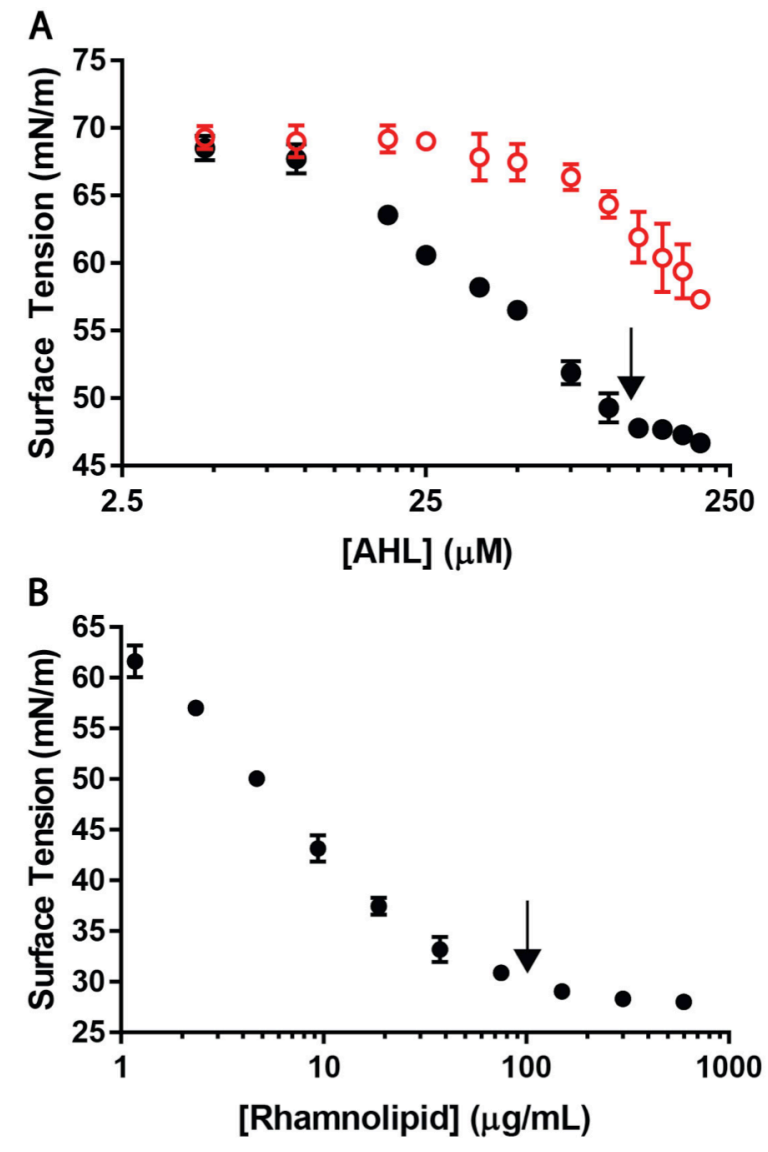

Figure S1: Surface tension measurements of (A) 3-oxo-C12-AHL (black, closed circles) and 3-oxo-C12-HS (red, open circles) or (B) rhamnolipid solutions at various concentrations. The data shown are the mean and a single standard deviation of three independent experiments. The breaks in the surface tension vs. concentration curves, which indicate the aggregation of 3-oxo-C12-AHL or rhamnolipid in solution, are indicated by arrows. 
3-Oxo-C12-AHL
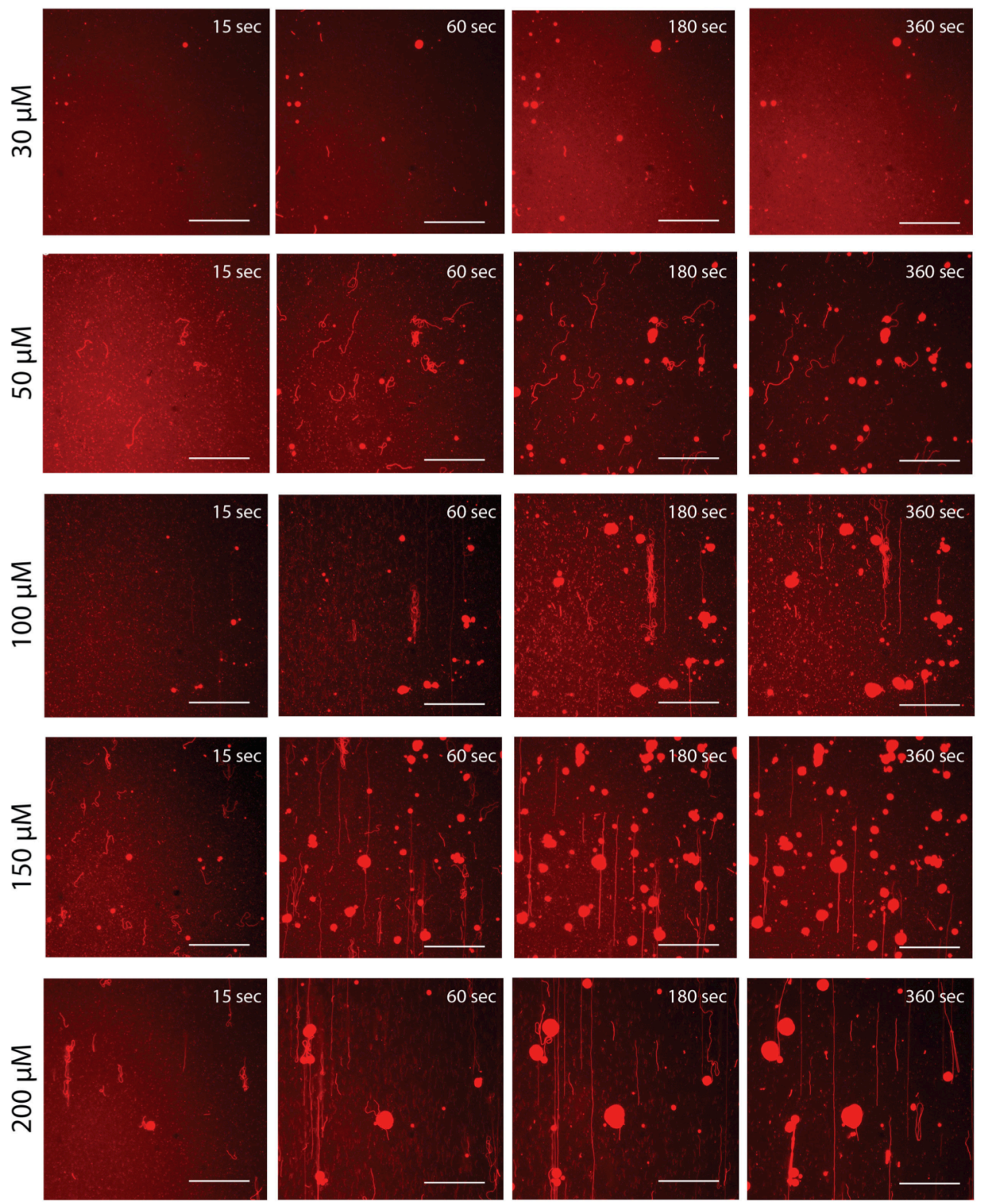

Figure S2a: Fluorescence micrographs showing the restructuring in DOPC SLBs after the introduction of 3-oxo-C12-AHL solutions at various concentrations over time. The images shown were taken at various time points indicated in the top-right corner of the images after the first membrane restructuring event was observed. The 360 -second images shown here are the same as those shown in Figure 4 of the main text. The concentration of 3-oxo-C12-AHL used in each experiment is shown in the column to the left of the images. The direction of flow in all the images was from the bottom to the top of the screen. The scale bars represent $30 \mu \mathrm{M}$. 


\section{3-oxo-C12-HS}
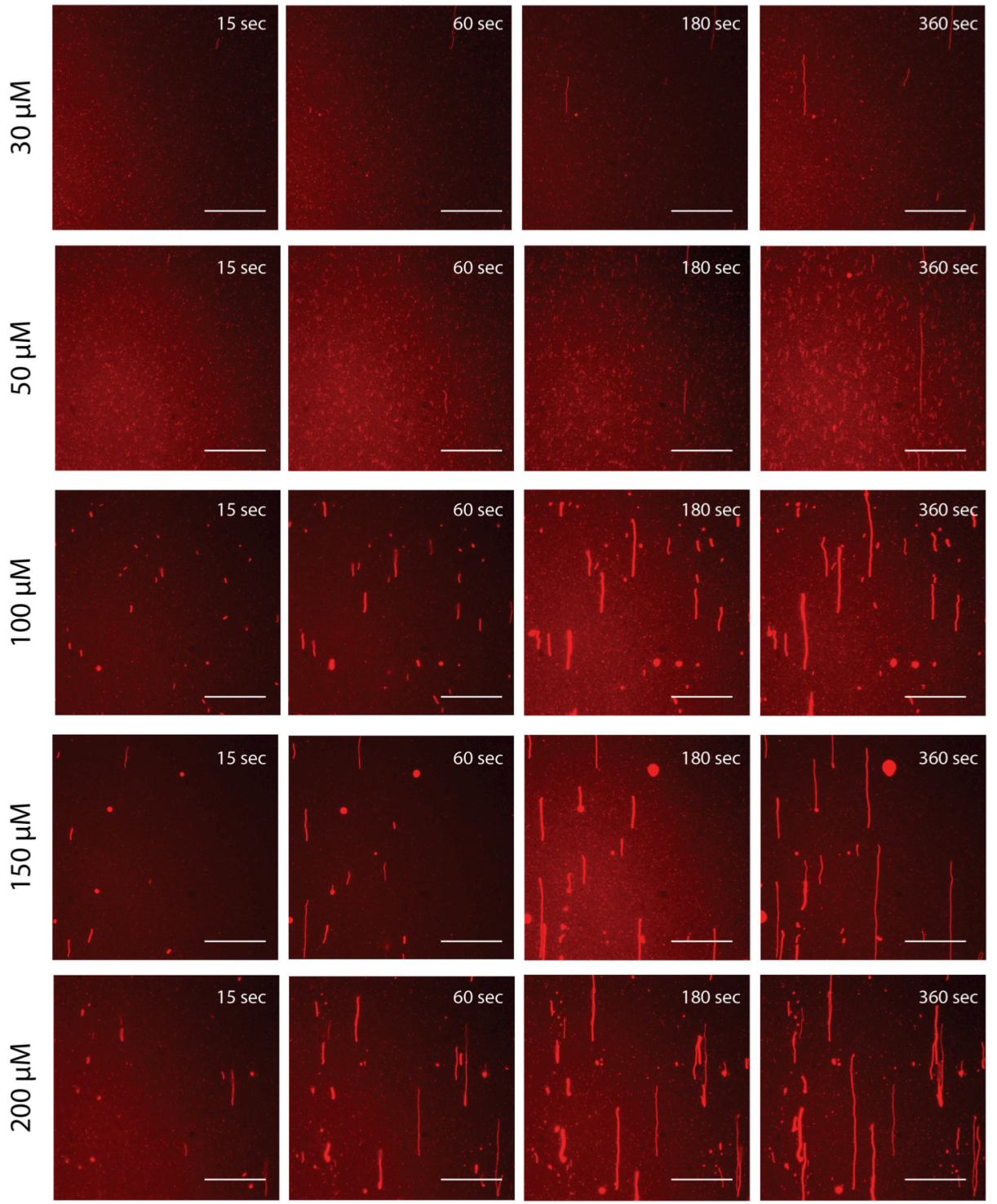

Figure S2b: Fluorescence micrographs showing the restructuring in DOPC SLBs after the introduction of 3-oxo-C12-HS solutions at various concentrations over time. The images shown were taken at various time points indicated in the top-right corner of the images after the first membrane restructuring event was observed. The 360 -second images shown here are the same as those shown in Figure 4 of the main text. The concentration of 3-oxo-C12-HS used in each experiment is shown in the column to the left of the images. The direction of flow in all the images was from the bottom to the top of the screen. The scale bars represent $30 \mu \mathrm{M}$. 

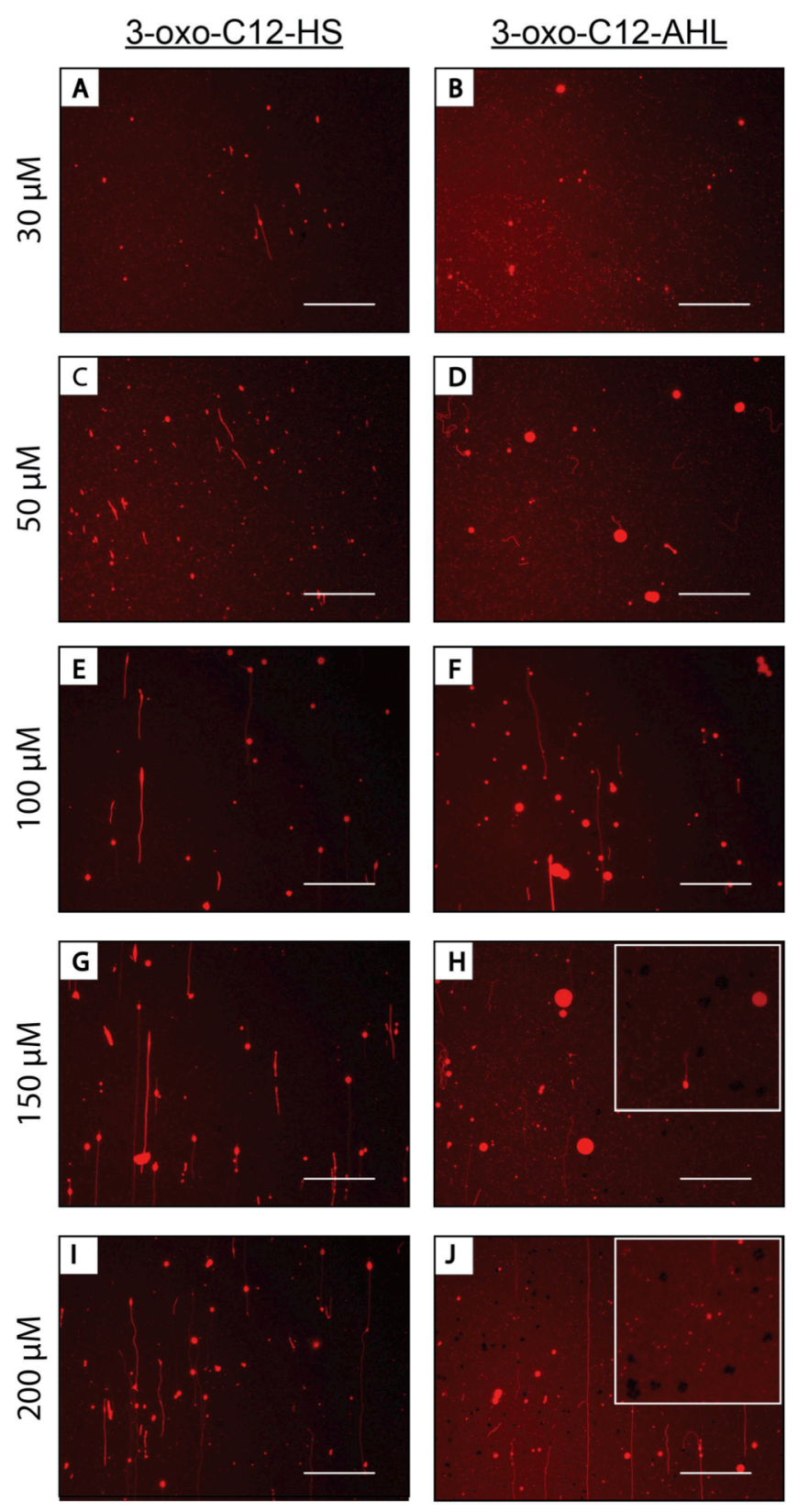

Figure S3: Representative top-down fluorescence micrographs showing DOPC SLBs that had previously been introduced to (A,C,E,G,I) 3-oxo-C12-HS or (B,D,F,H,J) 3-oxo-C12AHL solutions at (A,B) $30 \mu \mathrm{M},(\mathrm{C}, \mathrm{D}) 50 \mu \mathrm{M},(\mathrm{E}, \mathrm{F}) 100 \mu \mathrm{M},(\mathrm{G}, \mathrm{H}) 150 \mu \mathrm{M}$, or (I,J) $200 \mu \mathrm{M}$ after at least 8 minutes of washing under a continuous flow of buffer. The direction of flow in all images was from the bottom to the top of the image. Scale bars are $30 \mu \mathrm{m}$. The insets in $\mathrm{H}$ and $J$ show circular areas of decreased fluorescence observed in the bilayer after washing it with buffer. 

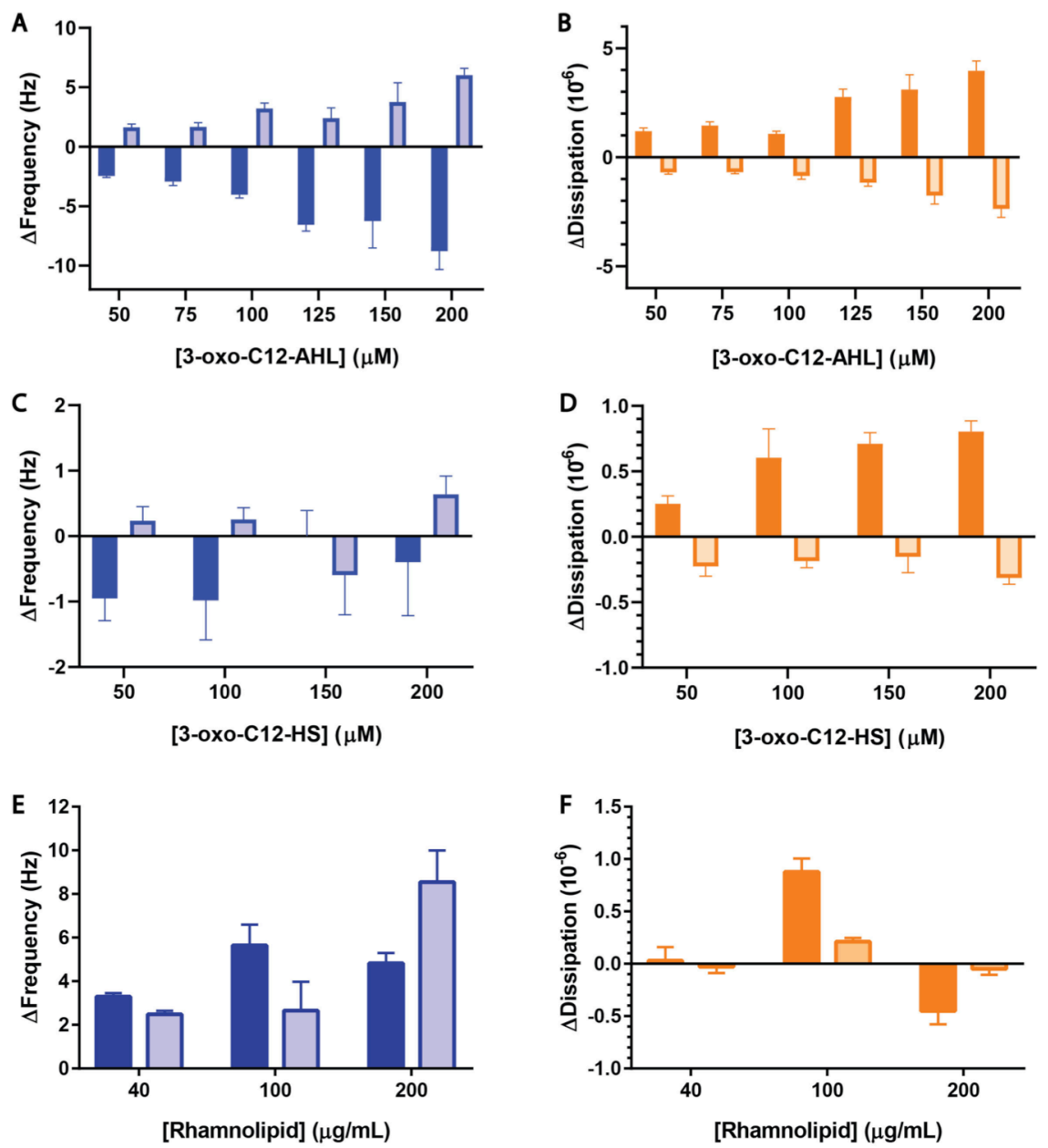

Figure S4: Changes in (A,C,E) frequency ( $\Delta$ Frequency; referred to as $\Delta \mathrm{f}$ in the main text for brevity) and (B,D,F) dissipation ( $\triangle$ Dissipation; referred to as $\Delta \mathrm{D}$ in the main text for brevity) for DOPC SLBs introduced to solutions of (A,B) 3-oxo-C12-AHL, (C,D) 3-oxo-C12-HS, and (E,F) rhamnolipid for 45 minutes (filled bars) or after 45 minutes of buffer wash (open bars) at various concentrations. The values shown are the means and a single standard deviation of three or four experiments $(n=3-4)$. 

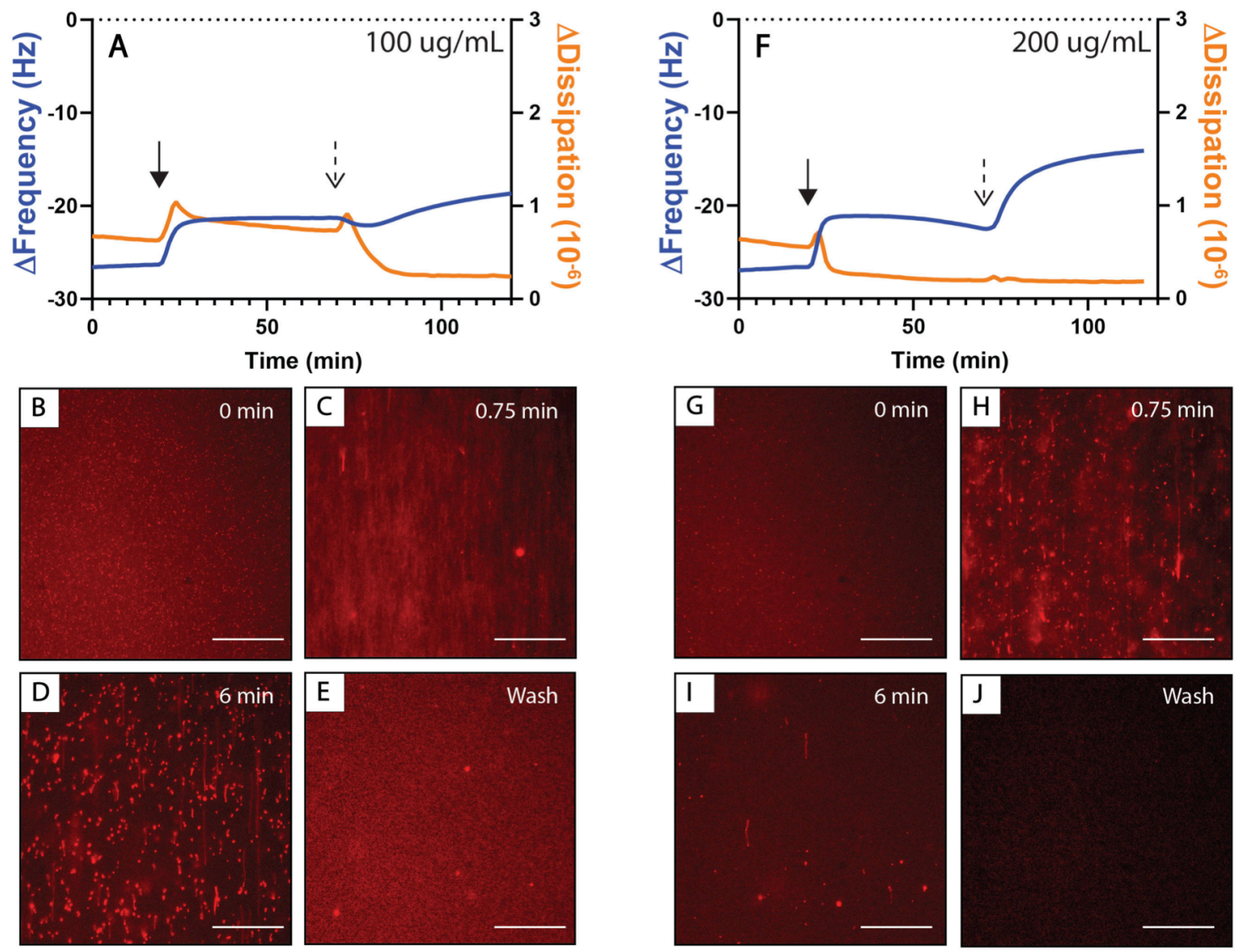

Figure S5: Measurements of membrane restructuring occurring in DOPC SLBs after introduction to (A-E) $100 \mu \mathrm{g} / \mathrm{mL}$ or (F-J) $200 \mu \mathrm{g} / \mathrm{mL}$ solutions of rhamnolipid. (A,F) QCM$\mathrm{D}$ measurements of bilayer reformations initiated by the introduction of rhamnolipid solutions with changes in frequency ( $\Delta$ Frequency, blue lines; referred to as $\Delta \mathrm{f}$ in the main text for brevity), and dissipation ( $\Delta$ Dissipation, orange lines; referred to as $\Delta \mathrm{D}$ in the main text for brevity) shown are representative of three or four independent experiments. The initial measurement values $(\mathrm{t}=0 \mathrm{~min})$ of these experiments correspond to DOPC SLBs on an $\mathrm{SiO}_{2}$ surface. Rhamnolipid solutions were introduced to the bilayer at the time indicated by the solid arrow and the buffer was introduced at the time indicated by the dashed arrow. (B-E,GJ) fluorescence micrographs of bilayer restructuring occurring at (B,G) $0 \mathrm{sec},(\mathrm{C}, \mathrm{H}) 45 \mathrm{sec}$, and (D,I) 6 min after the first bilayer restructuring events had been observed or (E,J) after at least 8 minutes of washing the bilayer with buffer. The direction of flow in all images was from the bottom to the top of the image. Scale bars are $30 \mu \mathrm{m}$. 


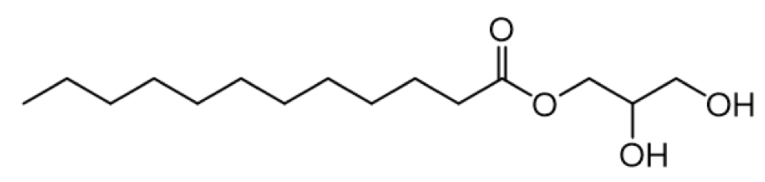

Glycerol monolaurate (GML)

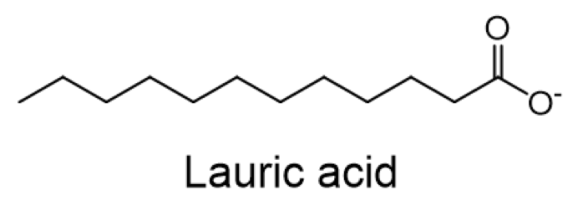

Figure S6: Chemical structures of glycerol monolaurate (GML) and lauric acid (LA). 

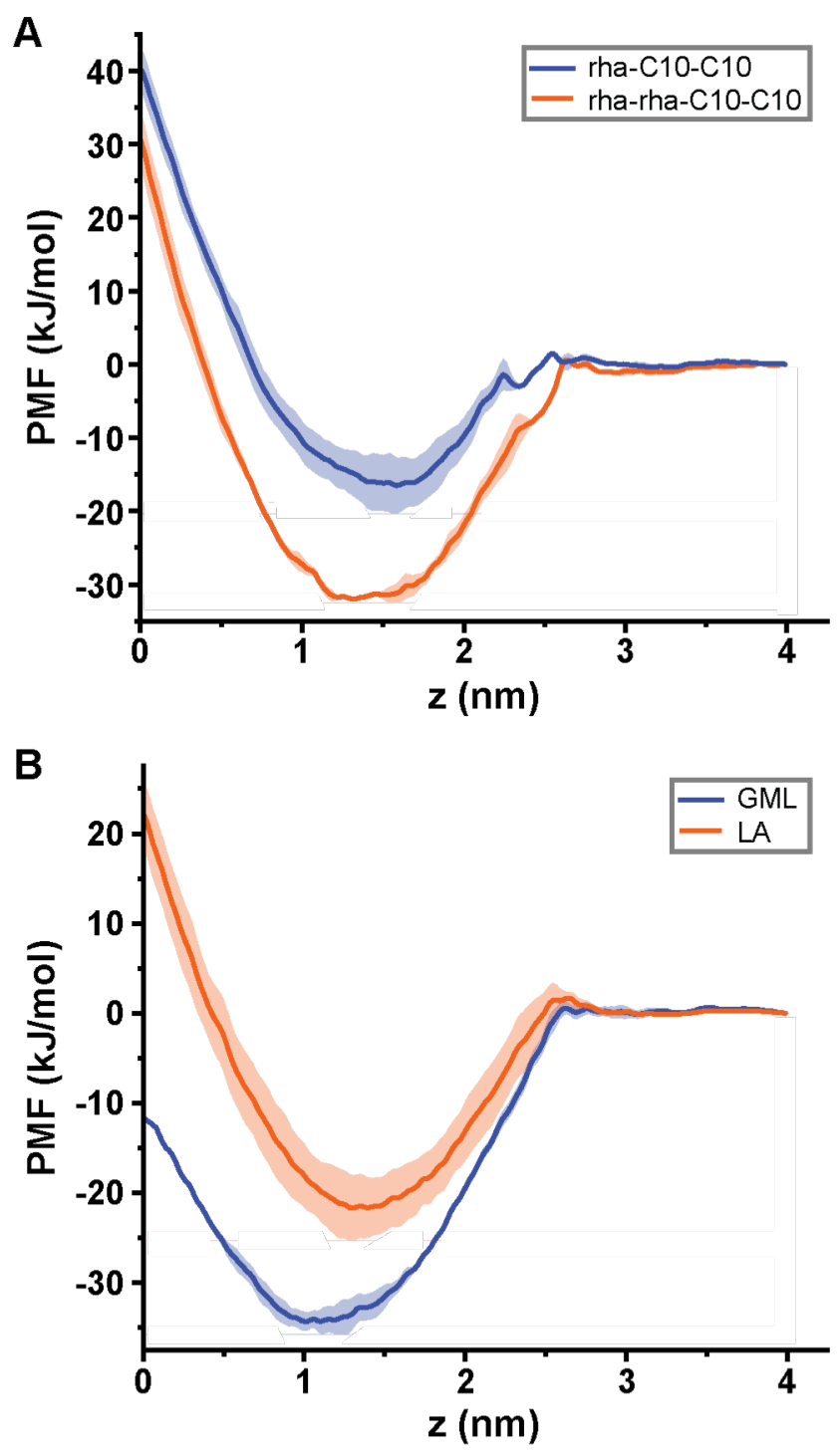

Figure S7: Potentials of mean force (PMFs) of (A) rha-C10-C10 (blue) and rha-rha-C10-C10 (orange), and (B) GML (blue) and LA (orange) as a function of the distance along the DOPC bilayer normal (z). 

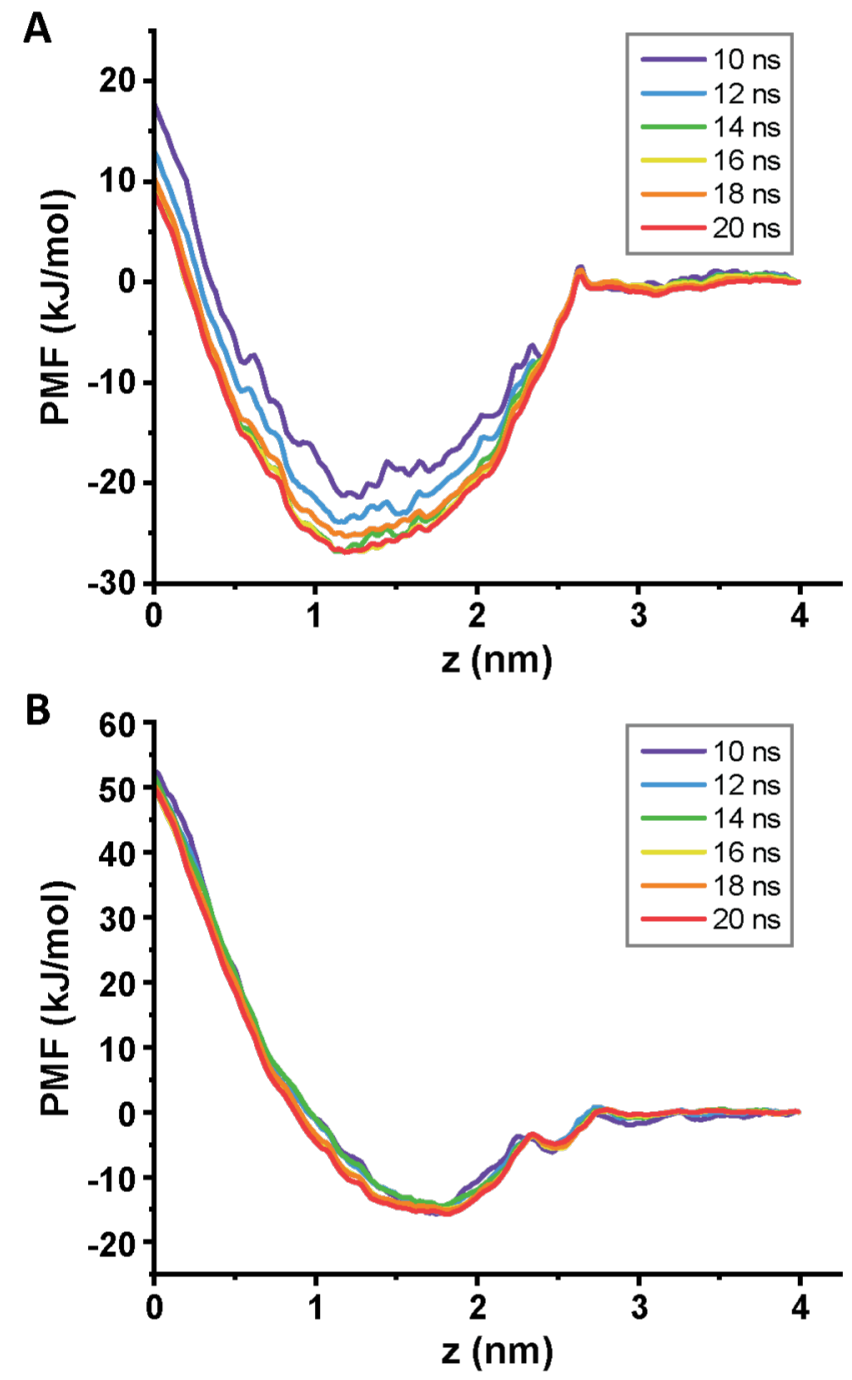

Figure S8: Representative convergence of the potentials of mean force (PMFs) computed using umbrella sampling as a function of the distance along the DOPC bilayer normal (z) for (A) 3-oxo-C12-AHL and (B) 3-oxo-C12-HS. 

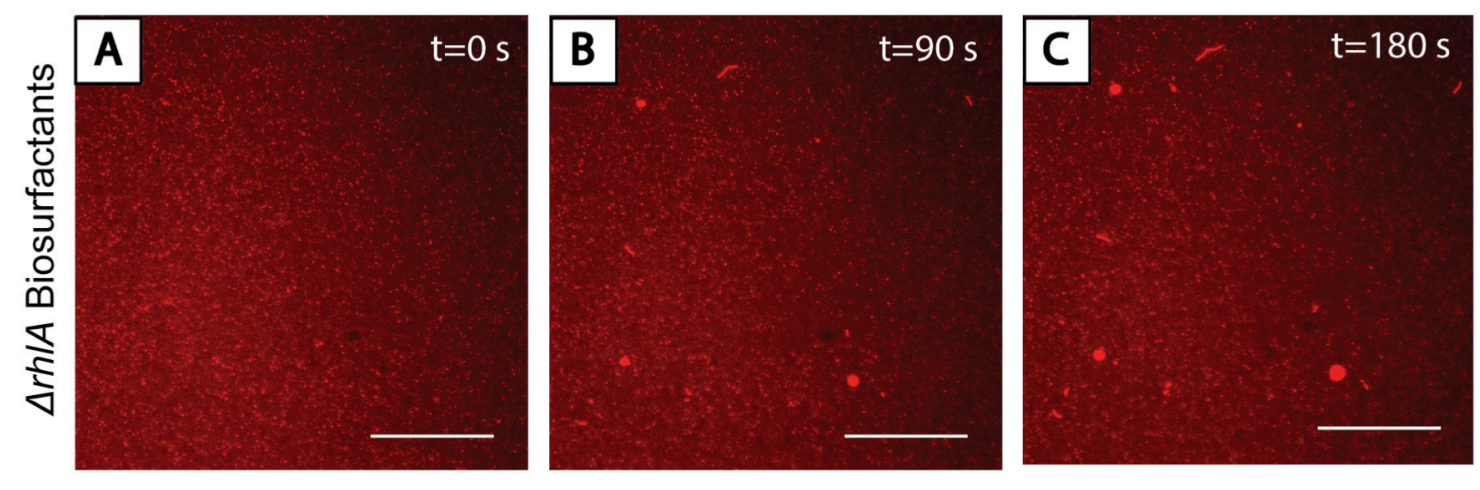

Figure S9: Representative top-down fluorescence micrographs of structural reformations occurring in fluorescently labeled DOPC SLBs after introduction to combinations of 3-oxoC12-AHL $(10 \mu \mathrm{M})$ and 3-oxo-C12-HS $(20 \mu \mathrm{M})$ that are representative of the concentrations of these molecules in $\triangle$ rhlA P. aeruginosa cultures grown for 24-hours. The micrographs were acquired at (A) 0 seconds, (B) 90 seconds, and (C) 180 seconds after the first membrane reformations were observed on the surface of the bilayer. The direction of flow in all images was from the bottom to the top of the panel. Scale bars are $30 \mu \mathrm{m}$.

Introduction of Biosurfactant Blends to SLBs. To confirm the bilayer responses observed when the supernatants of $\triangle r h l A P$. aeruginosa cultures were introduced to DOPC SLBs, we prepared a solution containing 3-oxo-C12-AHL $(10 \mu \mathrm{M})$ and 3-oxo-C12-HS $(20 \mu \mathrm{M})$ that is representative of the concentrations of these molecules in $\triangle r h l A$ P. aeruginosa cultures grown for 24-hours. ${ }^{1}$ When we introduced this solution to the SLB, we saw a combination of tubules and hemispherical caps appear on the surface of the bilayer (Figure S9), a behavior which resembled combinations of the behaviors observed when $30 \mu \mathrm{M}$ of 3-oxo-C12-AHL or 3-oxoC12-HS were introduced to the bilayer. These are also similar in type and extent to the reformations observed in our experiments with $\triangle r h l A$ supernatants in Figure 10(D-F) of the main text, suggesting that the 3-oxo-C12-AHL and 3-oxo-C12-HS components of these cultures are likely responsible for the remodeling observed. 
Synthesis Scheme of 3-oxo-C12-H Synthesis and Associated NMR Spectra. ${ }^{1} \mathrm{H}$ NMR (500 MHz, Methanol- $\left.d_{4}\right) \delta 4.35(\mathrm{dd}, J=8.4,5.1 \mathrm{~Hz}, 1 \mathrm{H}), 3.66(\mathrm{dd}, J=7.3,5.8 \mathrm{~Hz}, 2 \mathrm{H}), 2.67-2.45$ (m, 1H), 2.08 (dddd, $J=25.7,14.9,7.6,5.2 \mathrm{~Hz}, 2 \mathrm{H}), 1.85$ (ddt, $J=14.0,8.4,5.8 \mathrm{~Hz}, 1 \mathrm{H}), 1.66-$ $1.47(\mathrm{~m}, 2 \mathrm{H}), 1.42-1.17(\mathrm{~m}, 12 \mathrm{H}), 0.90(\mathrm{t}, J=6.9 \mathrm{~Hz}, 3 \mathrm{H}) .{ }^{13} \mathrm{C}$ NMR $(126 \mathrm{MHz}, \mathrm{MeOD}) \delta$ $205.75,177.74,167.51,58.66,52.37,42.47,35.25,31.65,29.32,29.19,29.17,29.06,29.01$, 28.77, 23.07, 22.32, 13.02. ESI-EMM: [M+Na] ${ }^{+}$calculated 338.1938; measured 338.1933.

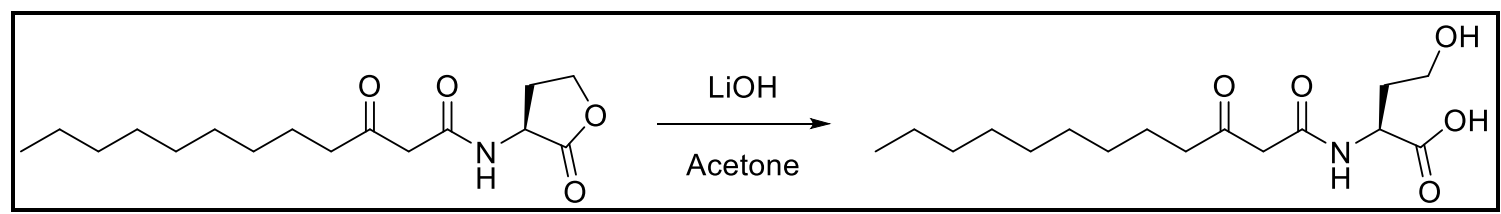

C1911181049_ro oddhl.10.fid

H1_standard.UW MeOD /home/dmanson/callisto dmanson 59

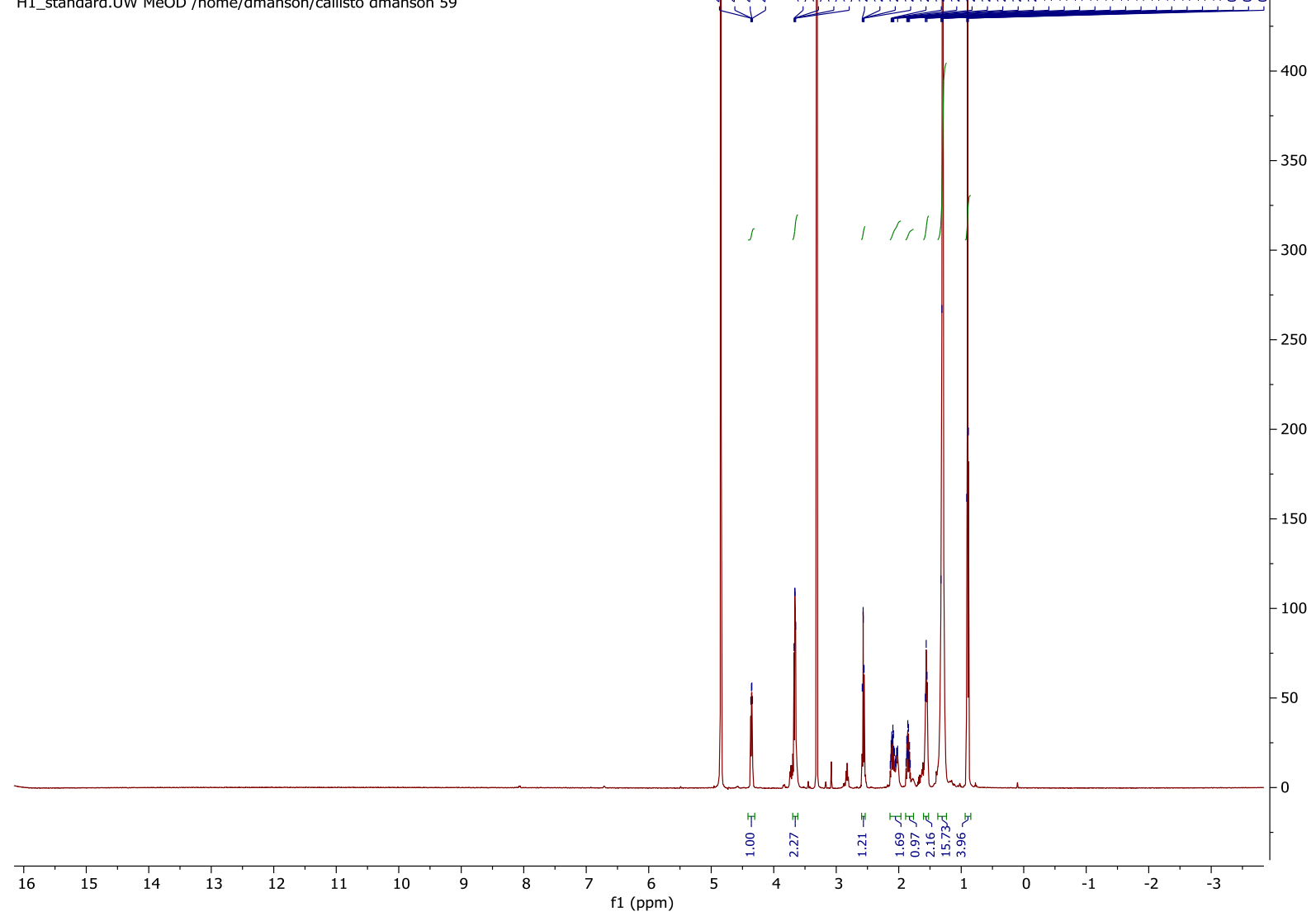




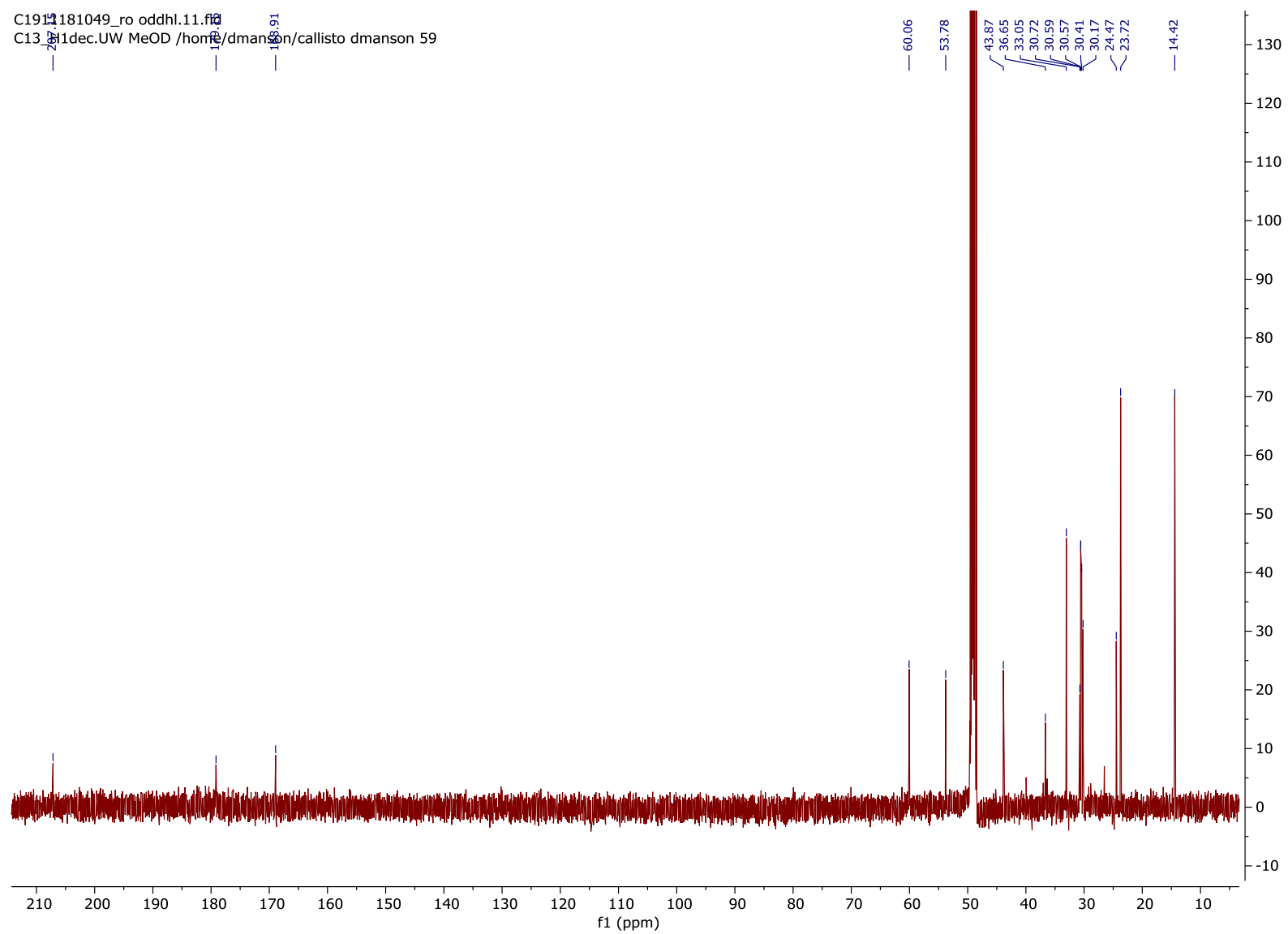

NMR Quantification of Phospholipids. To determine the concentration of phospholipids in stock solutions, quantitative ${ }^{31} \mathrm{P}\left[{ }^{1} \mathrm{H}\right]$ NMR spectroscopy was performed in $90 \% \mathrm{H}_{2} \mathrm{O}$ and $10 \%$ $\mathrm{D}_{2} \mathrm{O}$ using a Bruker Avance-400 spectrometer with a BBFO probe. A relaxation delay (D1) of $11 \mathrm{~s}$ was determined by inversion-recovery pulse sequence experiments. $890 \mu \mathrm{L}$ of a phospholipid vesicle sample in water was mixed with $10 \mu \mathrm{L}$ of Triton-X or rhamnolipid solution and $100 \mu \mathrm{L}$ of a $100 \mathrm{mM}$ triethylphosphine oxide internal standard solution in $\mathrm{D}_{2} \mathrm{O}$. All spectra were referenced relative to the phosphorus peak of the internal standard ( $\delta 65 \mathrm{ppm})$. Acquisition parameters were as follows: $\mathrm{PULPROG}=\mathrm{zgig} 30, \mathrm{D} 1=11 \mathrm{~s}, \mathrm{SW}=405 \mathrm{ppm}, \mathrm{O} 2 \mathrm{P}=3.75 \mathrm{ppm}$, NS=64, $\mathrm{DS}=4, \mathrm{LB}=1$. 


\section{References}

1. Ortiz, B. J.; Boursier, M. E.; Barrett, K. L.; Manson, D. E.; Amador-Noguez, D.;

Abbott, N. L.; Blackwell, H. E.; Lynn, D. M., Liquid Crystal Emulsions That Intercept and Report on Bacterial Quorum Sensing. ACS Appl. Mater. Interfaces 2020, 12 (26), 29056-29065. 